\title{
Band-limited Green's Functions for Quantitative Evaluation of Acoustic Emission using the Finite Element Method
}

\author{
William P. Leser* \\ NASA Langley Research Center/North Carolina State University, Hampton, VA 23681, U.S.A. \\ Fuh-Gwo Yuan ${ }^{\dagger}$ \\ North Carolina State University/National Institute of Aerospace, Hampton, VA 23666, U.S.A. \\ John A. Newman \\ NASA Langley Research Center, Hampton, VA, 23681, U.S.A.
}

\begin{abstract}
A method of numerically estimating dynamic Green's functions using the finite element method is proposed. These Green's functions are accurate in a limited frequency range dependent on the mesh size used to generate them. This range can often match or exceed the frequency sensitivity of the traditional acoustic emission sensors. An algorithm is also developed to characterize an acoustic emission source by obtaining information about its strength and temporal dependence. This information can then be used to reproduce the source in a finite element model for further analysis. Numerical examples are presented that demonstrate the ability of the band-limited Green's functions approach to determine the moment tensor coefficients of several reference signals to within seven percent, as well as accurately reproduce the source-time function.
\end{abstract}

\section{Nomenclature}

$G$ Dynamic Green's function

$\vec{x}, \vec{\xi} \quad$ Location vectors

$t \quad$ Time

$C_{i j k l}$ Elastic stiffness tensor

$u_{i} \quad$ Displacement vector

$M \quad$ Seismic moment tensor

$m_{i} \quad$ Moment tensor coefficient

* Convolution operator

$\lambda \quad$ Bulk modulus

$\mu \quad$ Shear modulus

\section{Introduction}

Acoustic emissions are transient elastic waves resulting from the sudden release of strain energy at the onset and/or progression of material damage or degradation. These elastic waves propagate through the

\footnotetext{
*Student Trainee (Engineering), Durability, Damage Tolerance, and Reliability Branch (D309), 2 West Reid Street, M/S 188E, Hampton, VA 23681-2199, Student Member.

${ }^{\dagger}$ Professor, Department of Mechanical and Aerospace Engineering, Engineering Bldg III, Campus Box 7910, 911 Oval Drive, Raleigh, NC 27695-7910, Associate Fellow.

${ }^{\ddagger}$ Materials Engineer, Durability, Damage Tolerance, and Reliability Branch (D309), 2 West Reid Street, M/S 188E, Hampton, VA 23681-2199.
} 
medium where they can be detected by sensors mounted remotely on the surface. Acoustic emission (AE) monitoring can be used as a viable nondestructive method for the real time monitoring of damage progression in a structure while in service. AE differs from other nondestructive evaluation (NDE) methods in that it monitors generally irreversible processes and therefore can only be used to detect the evolution of damage as it occurs. The advantage of $\mathrm{AE}$ is that it only requires a small set of passive transducers to monitor a structure. ${ }^{1}$

$\mathrm{AE}$ analysis has typically been considered a qualitative exercise, where correlations between experimental results and $\mathrm{AE}$ signal characteristics are observed and used in pattern recognition schemes to draw conclusions during future $\mathrm{AE}$ monitoring applications. ${ }^{1}$ The limitation with this approach is that there is no underlying physics identified between the experimental results and the AE signal characteristics. Different sources producing AE signals with similar characteristics may lead to unsubstantiated conclusions being drawn. To overcome this shortcoming, methods of quantitative AE evaluation have been developed to study the details of the AE source and resulting waves. These methods will allow more quantitative conclusions to be drawn about the correlation between damage and the resulting AE signals as well as providing for an improved ability to distinguish between sources that share similar characteristics. ${ }^{2,3}$

\section{Theory}

\section{II.A. Theoretical Representation of an Acoustic Emission Source}

To extract quantitative data from an acoustic emission event, the source of the emission must be able to be mathematically modeled in some fashion. Scruby has shown that an acoustic emission source can be represented as a point source with two main assumptions. ${ }^{2}$ First, it is assumed the source is internal and self-equilibrating. This means that every force or couple that makes up the source must be a force dipole or double couple. Second, all forces that make up the source occur simultaneously, such that they all share the same time history. The displacement response on the surface of the body due to the point source can then be calculated as follows.

Consider a force, $h_{j}(\vec{\xi}, t)$, applied in the $j$ th direction to a body at position $\vec{\xi}$ as shown in figure 1 . The displacement at location $\vec{x}$ in the $i$ th direction, $u_{i}(\vec{x}, t)$, can be calculated as

$$
u_{i}(\vec{x}, t)=h_{j}(\vec{\xi}, t) * G_{i j}(\vec{x}, t, \vec{\xi})
$$

where $*$ denotes convolution, vectors are represented with an arrow, and $G_{i j}(\vec{x}, t, \vec{\xi})$ is the Green's function, defined as the displacement response at $\vec{x}$ in the $i$ th direction due to an impulse load at $\vec{\xi}$ in the $j$ th direction. ${ }^{3}$

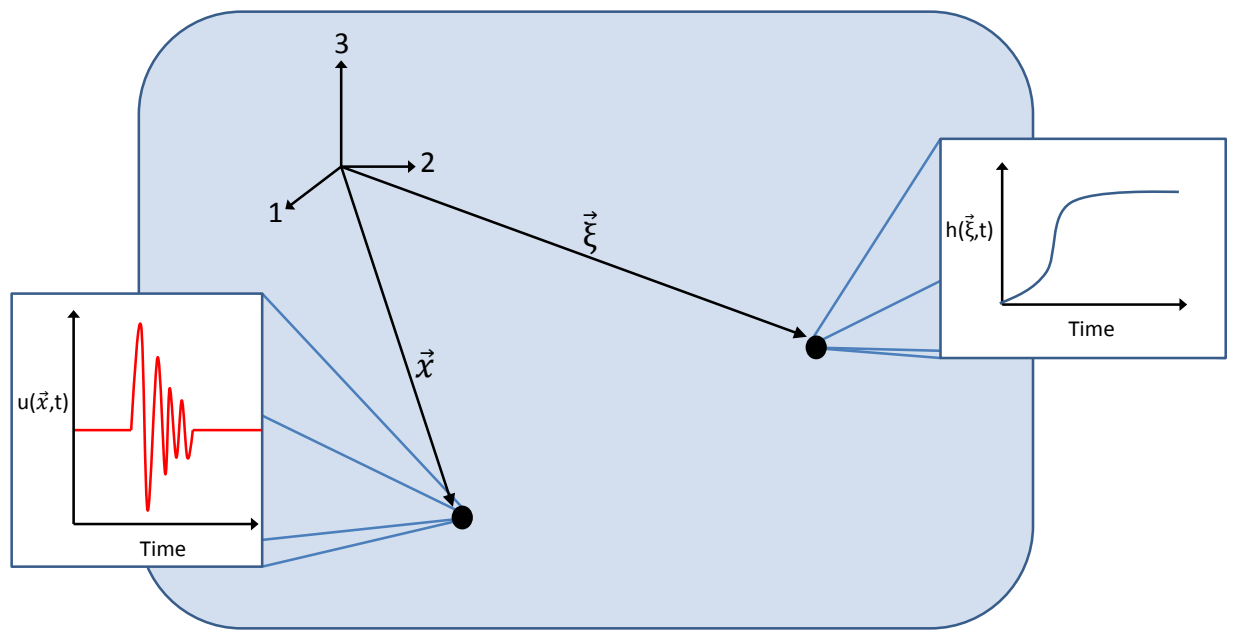

Figure 1. Point source and sensor locations in a general elastic body.

In order to meet the assumptions of an internal, self-equilibrating source, the force, $h_{j}(\vec{\xi}, t)$ is replaced with a moment tensor, M, containing the strengths of the system of dipole forces representing the AE source, 
the components of which are denoted as $m_{j k}(\vec{\xi})$. The equivalent force dipole or couple for each moment tensor component is shown in figure 2. All of the forces that make up $\mathbf{M}$ act with the same temporal behavior, $f(t)$. Thus, Eq. (1) becomes

$$
u_{i}(\vec{x}, t)=\sum_{j=1}^{3} \sum_{k=1}^{3} m_{j k}(\vec{\xi}) f(t) * G_{i j, k}(\vec{x}, t, \vec{\xi})
$$

where $G_{i j, k}(\vec{x}, t, \vec{\xi})$ is the Green's function of a dipole source, defined as the impulse displacement response in the $i$ th direction at $\vec{x}$ due to a dipole impulse force in the $j$ th direction at location $\vec{\xi}$, where the impulse forces that make up the dipole are separated in the $k$ th direction.
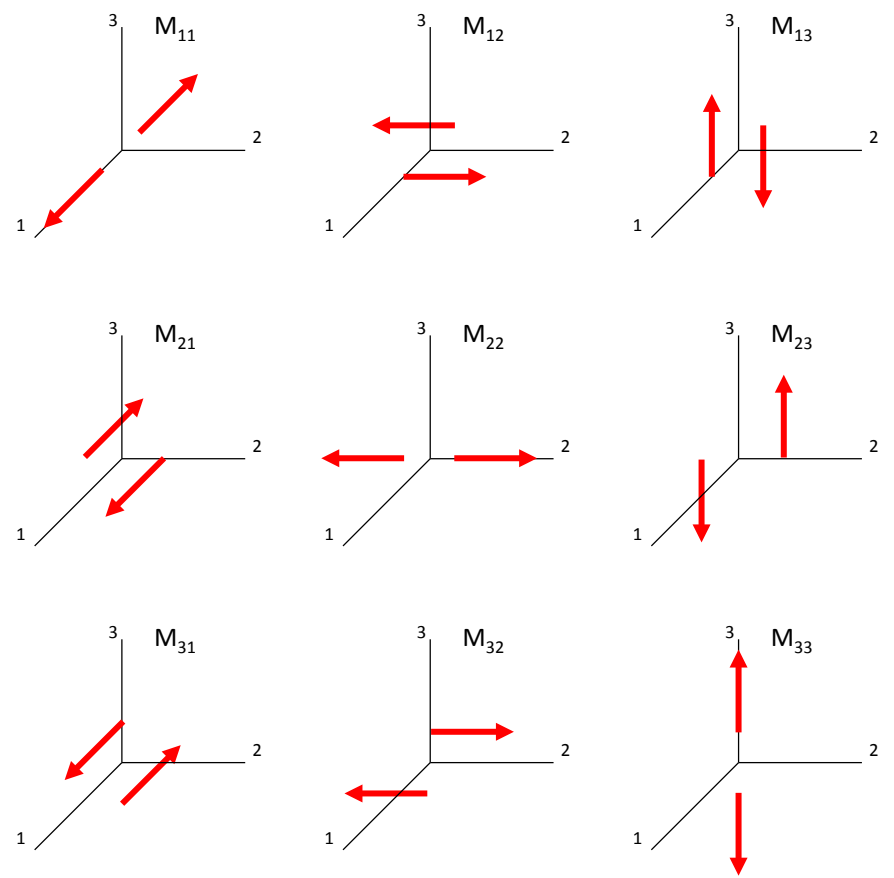

Figure 2. Force dipoles and double couples corresponding to the moment tensor.

The values of the moment tensor coefficients for a displacement discontinuity can be determined for homogeneous materials according to the relation ${ }^{1,3}$

$$
m_{i j}=C_{i j k l} b_{k} d A_{l}
$$

where $C_{i j k l}$ is the elastic stiffness tensor. For an isotropic solid, $C_{i j k l}$ is given as

$$
C_{i j k l}=\lambda \delta_{i j} \delta_{k l}+\mu\left(\delta_{i k} \delta_{i l}+\delta_{j l} \delta_{j k}\right)
$$

where $\delta_{i j}$ is the Kronecker delta $\left(\delta_{i j}=1\right.$ if and only if $i=j$ ), and $\lambda$ and $\mu$ are Lame's constants.

As an example, consider a crack with faces each having surface area $d A$ in the $x_{1} x_{2}$ plane that opens in the $x_{3}$ direction. The equivalent body forces are found by substituting $l=k=3$ into Eq. (3) and Eq. (4) and the components of the resulting moment tensor are:

$$
\begin{aligned}
m_{j k} & =\lambda \delta i j+\mu\left(\delta_{i 3} \delta_{i 3}+\delta_{j 3} \delta_{j 3}\right) b d A \\
\mathbf{M} & =\left[\begin{array}{ccc}
\lambda & 0 & 0 \\
0 & \lambda & 0 \\
0 & 0 & \lambda+2 \mu
\end{array}\right] b d A
\end{aligned}
$$




\section{II.B. Finite Element Representation of an Acoustic Emission Source}

The finite element method (FEM) can be used to model acoustic emission wave forms. FEM has several distinct advantages over traditional analytical approaches to modeling AE waveforms. Analytical approaches are typically limited to very simple geometry, i.e. thin, infinite plates or half spaces. In contrast, FEM can handle complex geometries, including boundary reflections. The main disadvantage of using FEM is the computational time required. The frequency range that can be modeled is determined by the element size used (smaller elements are needed to capture higher frequencies), so there is a trade off that must be made between the frequency range of the model and the computational time required. ${ }^{4}$

AE sources cannot be modeled as a point source when using finite elements. Therefore, sources are approximated as point sources by body forces that are applied over some finite area. Hamstad, et. al. have developed a set of criteria for the convergence of a finite element model of an acoustic emission source based on the maximum frequency of interest, $f_{\max }$, which is inversely proportional to the minimum wavelength of interest, $\lambda_{\min } \cdot{ }^{4}$

$$
\lambda_{\min }=\frac{c_{T}}{f_{\max }}
$$

where $c_{T}$ is the transverse, or shear, wave velocity in the material. Several relations were developed between the minimum wavelength, $\lambda_{\text {min }}$, source size, $s$, finite element mesh size, $M S$, and the distance between the source and the transducer, $D$. Convergence was measured by the normalized amplitude of results with different parameters. The following criteria were found for convergence of peak amplitudes in modeled signals to within $5 \%,{ }^{4}$

$$
\begin{aligned}
\frac{\lambda_{\min }}{s} & \geq 2 \\
\frac{\lambda_{\min }}{M S} & \geq 15 \\
\frac{D}{s} & \geq 7
\end{aligned}
$$

In this study, all FEM calculations are made using the commercially available software $A B A Q U S^{T M} .^{5}$ A comparison between an analytical solution, found in the literature, for a buried dipole in a steel plate and the results of a finite element simulation is shown in figure $3{ }^{4}$ It can be seen that the FEM solution accurately captures all of the features of the analytical solution. Therefore, FEM will be used to calculate all reference solutions for this study. For the comparison, a vertical force dipole of strength $0.3 \mathrm{~N} \cdot \mathrm{mm}$ was located at the center of an axi-symmetric model of a $25 \mathrm{~mm}$ thick steel disk. The sensor was located $50 \mathrm{~mm}$ from the epicenter as shown in figure 4. FEM parameters for all simulations done in this study are shown in table 1.

Table 1. Model parameters for FEM analysis.

\begin{tabular}{lcccccc} 
& Mesh $(\mu \mathrm{m})$ & Elements & Dipole Spacing $(\mu \mathrm{m})^{*}$ & $\mathrm{E}(\mathrm{GPa})$ & $\nu$ & $\rho\left(\mathrm{kg} / \mathrm{m}^{3}\right)$ \\
\hline Figure 4: & 50 & 4 node axi-symmetric & 50 & 209 & 0.29 & 7800 \\
Figure 5: & 50 & 4 node axi-symmetric & 50 & 73 & 0.30 & 2770 \\
Figure 12: & 50 & 8 node brick & 50 & 73 & 0.30 & 2770 \\
Figure 14: & $100^{* *}$ & 10 node tet & 50 & 73 & 0.30 & 2770 \\
\hline
\end{tabular}

*All sources are $150 \mu m$ in size. $50 \mu m$ for each dipole with $50 \mu m$ separating them.

** Mesh size at source equal to $50 \mu m$, constrained to a maximum size of $100 \mu m$ everywhere else.

\section{II.C. Calculation of the Dynamic Green's Function}

Central to the characterization of AE events, is the calculation of the dynamic Green's function between the source location and each sensor. Analytical solutions to Green's functions are difficult if not impossible to determine for all but the simplest of geometries, including infinite plates and half-spaces. ${ }^{2}$ It is proposed that numerical approximations of the dynamic Green's functions for a source/sensor pair can be calculated using FEM. The numerical Green's functions calculated in this manner are not true Green's functions. A 


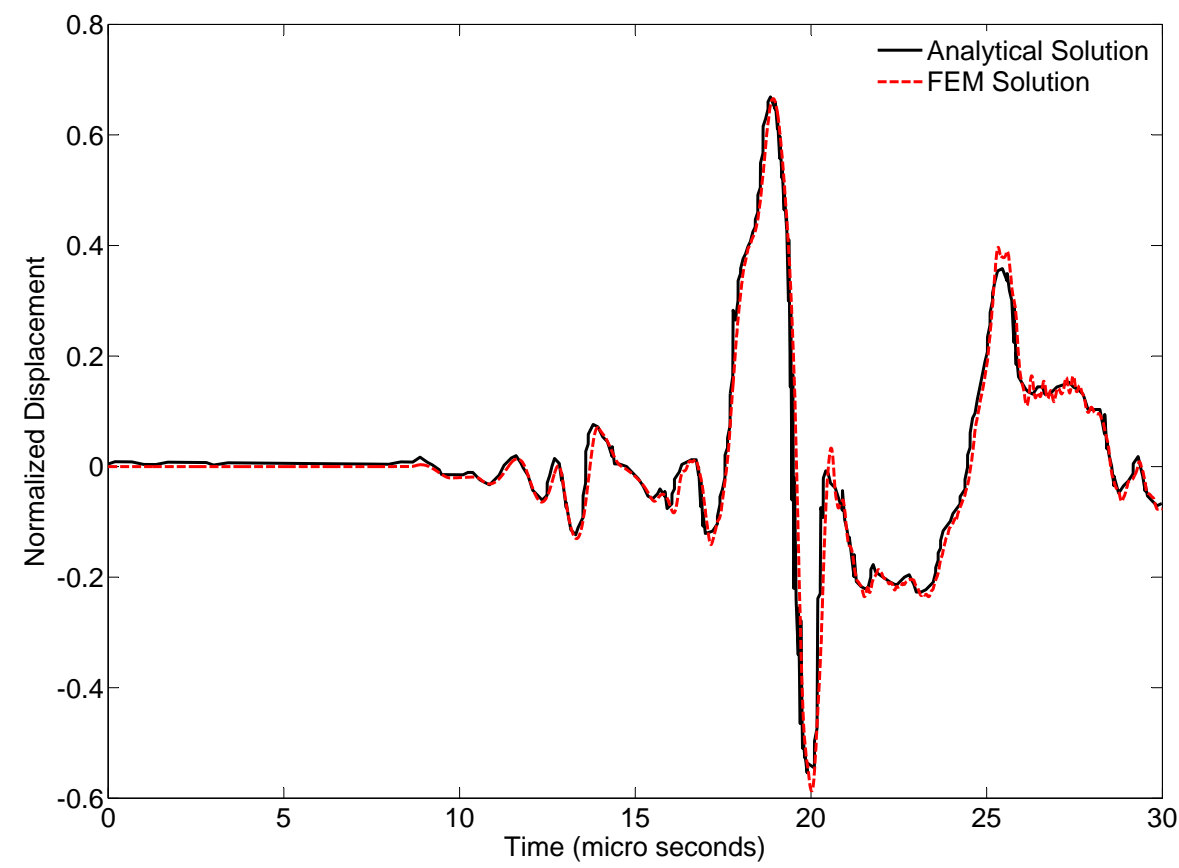

Figure 3. Comparison between a finite element generated AE signal and an analytically derived signal in a $25 \mathrm{~mm}$ thick steel disk from Hamstad (1999).

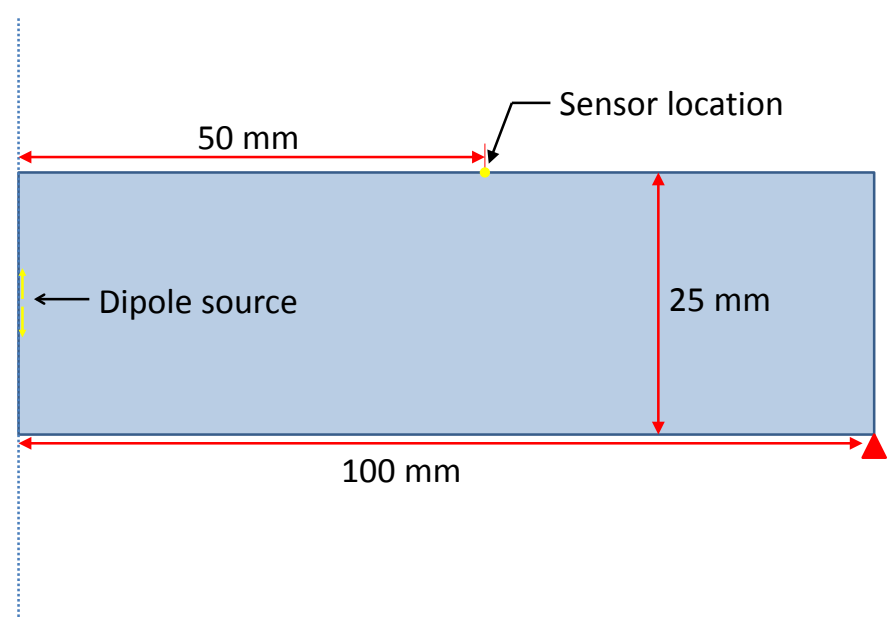

Figure 4. Schematic of geometry and boundary conditions for axi-symmetric finite element model used for comparison with analytical solution.

true Green's function will represent the impulse response of the system at all frequencies. The numerical approximations of the Green's function generated by FEM however, are band-limited based on the mesh size used to generate them. Therefore, any AE signal modeled using a band-limited Green's function will not converge at higher frequencies. However, the typical AE sensor loses sensitivity in the $1-2 \mathrm{MHz}$ range. ${ }^{6}$ For the quantitative analysis of experimental $\mathrm{AE}$ data, the frequency range of the band-limited Green's function only needs to match that of the sensor being used.

For convenience, a modification is made to Eq. (2). First, it is assumed that the sensors used in all experiments are only sensitive to displacement normal to the surface of the body they are monitoring. Therefore, the index $i$ in Eq. (2) can be dropped as it is assumed that the displacement, $u$, and Green's 
function, $G$, always reference the displacement normal to the surface. Second, due to the symmetry of linear elasticity, an index transformation can be applied to $m_{j k}$ and $G_{j, k}$ such that,

$$
\begin{aligned}
m_{11} & =m_{1} \\
m_{22} & =m_{2} \\
m_{33} & =m_{3} \\
m_{12} \& m_{21} & =m_{4} \\
m_{13} \& m_{31} & =m_{5} \\
m_{23} \& m_{32} & =m_{6} \\
G_{1,1} & =G_{1} \\
G_{2,2} & =G_{2} \\
G_{3,3} & =G_{3} \\
G_{1,2} \& G_{2,1} & =G_{4} \\
G_{1,3} \& G_{3,1} & =G_{5} \\
G_{2,3} \& G_{3,2} & =G_{6}
\end{aligned}
$$

thus, Eq. (2) becomes

$$
u^{r}=\sum_{j=1}^{6} m_{j} f(t) * G_{j}^{r} \quad r=1 \ldots n
$$

where $n$ is the number of sensors.

A finite element model of the specimen is then generated and run six times in order to obtain a set of displacements related to each component of the moment tensor. Each run uses a different dipole or double couple of a known strength and time dependence located at the position of the AE source. For example, for the first run set $m_{1}=1$ and $m_{2-6}=0$. For the second run, $m_{2}=1$ and $m_{1}=m_{3-6}=0$ and so on. Displacement output normal to the surface is recorded at each sensor location. The displacement output corresponds to $u^{r}$ and the prescribed dipole strength and time dependence correspond to $m_{j}$ and $f(t)$ in Eq. (11). Then, $G_{j}$ is found through the deconvolution of $m_{j} f(t)$ from $u^{r}$.

For the present study, a least squares approach to deconvolution is used. Let $j=1$ and $m_{1}=1$. The convolution equation representing the displacement response due to a dipole in the $x_{1}$ direction is given by

$$
u(t)=f(t) * G_{1}(t)
$$

and can be rewritten in matrix form $\mathrm{as}^{3}$

$$
\left[\begin{array}{c}
u_{1} \\
u_{2} \\
\vdots \\
u_{m}
\end{array}\right]=\left[\begin{array}{ccccc}
f_{1} & 0 & 0 & \ldots & 0 \\
f_{2} & f_{1} & 0 & \ldots & 0 \\
\vdots & \vdots & \vdots & \ddots & \vdots \\
f_{m} & f_{m-1} & f_{m-2} & \ldots & f_{m-n+1}
\end{array}\right]\left[\begin{array}{c}
G_{1_{1}} \\
G_{1_{2}} \\
\vdots \\
G_{1_{n}}
\end{array}\right]
$$

where $m$ and $n$ are the number of the data points in the displacement and Green's function vectors. The unknown Green's function $G_{1}(t)$ is then found by solving Eq. (13) using the conjugate gradient method. ${ }^{7}$ Deconvolution is extremely sensitive to error, especially in the higher frequency range. Therefore, it is important that the tolerances are not set too small during the deconvolution process. Some trial and error may be required to obtain optimal performance. ${ }^{8}$

\section{II.D. Calculation of the Moment Tensor and Source-Time Dependence}

Once the band-limited Green's functions have been found for each component of the moment tensor, the moment tensor and source time dependence can be recovered from Eq. (11). This is accomplished by making an initial guess for $f(t)$ and calculating the values of $m_{j}$ using the conjugate gradient method as follows. 
$f(t)$,

Let $w_{j}$ represent the convolution of each Green's function with the initial guess of the source time function

$$
w_{j}=f(t) * G_{j}
$$

Convolution in the time domain is equivalent to multiplication in the frequency domain, so to calculate $w_{j}$, $f(t)$ and $G_{j}$ are transformed into the frequency domain using the Fourier transform, multiplied together, and then returned to the time domain via the inverse Fourier transform. Substituting Eq. (14) into Eq. (11) gives

$$
u^{r}=\sum_{j=1}^{6} m_{j} w_{j}^{r} \quad r=1 \ldots n
$$

Let $[u(t)]$ denote the time integral of function $u(t)$ such that

$$
[u(t)]=\int_{0}^{t} u(t) d t
$$

Integrating both sides of Eq. (15) yields

$$
\left[u^{r}\right]=m_{1}\left[w_{1}^{r}\right]+m_{2}\left[w_{2}^{r}\right]+m_{3}\left[w_{3}^{r}\right]+m_{4}\left[w_{4}^{r}\right]+m_{5}\left[w_{5}^{r}\right]+m_{6}\left[w_{6}^{r}\right] \quad r=1 \ldots n
$$

which can be written in matrix form as

$$
\left[\begin{array}{c}
{\left[u^{1}\right]} \\
{\left[u^{2}\right]} \\
\vdots \\
{\left[u^{n}\right]}
\end{array}\right]=\left[\begin{array}{cccc}
{\left[w_{1}^{1}\right]} & {\left[w_{2}^{1}\right]} & \ldots & {\left[w_{6}^{1}\right]} \\
{\left[w_{1}^{2}\right]} & {\left[w_{2}^{2}\right]} & \ldots & {\left[w_{6}^{2}\right]} \\
\vdots & \vdots & \ddots & \vdots \\
{\left[w_{1}^{n}\right]} & {\left[w_{2}^{n}\right]} & \ldots & {\left[w_{6}^{n}\right]}
\end{array}\right]\left[\begin{array}{c}
m_{1} \\
m_{2} \\
\vdots \\
m_{6}
\end{array}\right]
$$

Eq. (18) can then be solved using the conjugate gradient method. The resulting values of $m_{j}$ are then used to deconvolve a refined approximation of $f(t)$ from Eq. (11) using the same method applied in Eq. (13) that was used to find the band-limited Green's functions. The process is repeated until the values of $m_{j}$ and $f(t)$ converge.

\section{Numerical Examples}

\section{III.A. Estimation of the Dynamic Green's Function}

A band-limited Green's function was calculated for a buried dipole at the mid-plane of a $10 \mathrm{~mm}$ thick aluminum disk of radius $50 \mathrm{~mm}$. The sensor was located $25 \mathrm{~mm}$ from the center of the plate as shown in figure 5. The Green's function was then used with Eq. (11) to calculate the response from 3 different dipole sources. The moment tensor (or dipole strength) used for all 3 sources was

$$
M=\left[\begin{array}{ccc}
0 & 0 & 0 \\
0 & 0.3 & 0 \\
0 & 0 & 0
\end{array}\right]
$$

with the plate laying in the $x_{1} x_{3}$ plane. The source-time dependence functions for each source are shown in figure 6. These functions were chosen arbitrarily to test the Green's function response to source-time functions with differing behavior. In case 1, the dipole forces reach their maximum value after $1.0 \mu s$ and then persist at a constant value. Case 2 models an impulse with a duration of $1.0 \mu s$. The dipole reaches its maximum strength at $0.5 \mu \mathrm{s}$ and then returns to 0 at $1.0 \mu \mathrm{s}$. In case 3 , the dipole reaches full strength in $0.5 \mu s$ and then reverses direction and reaches full strength again at $1.5 \mu s$ and remains constant.

To check the accuracy of the numerically estimated Green's function, the three AE signals calculated using Eq. (11) are then compared with reference solutions calculated via direct FEM simulations in figures 7 (case 1), 8 (case 2) and 9 (case 3). The results show good agreement between the signals calculated with the band-limited Green's function and the signals generated directly by FEM. The maximum difference between the two signals is only $0.4 \%$. This demonstrates that the FEM generated Green's function accurately captures the impulse response of the plate. 


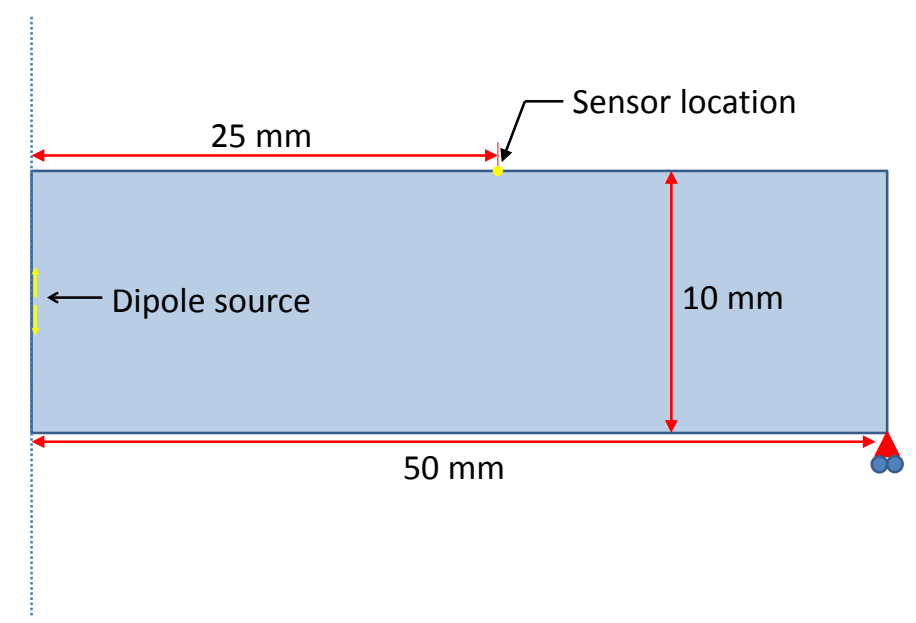

Figure 5. Schematic of geometry and boundary conditions for axi-symmetric finite element model used for numerical estimation of the Green's function for a buried dipole source.
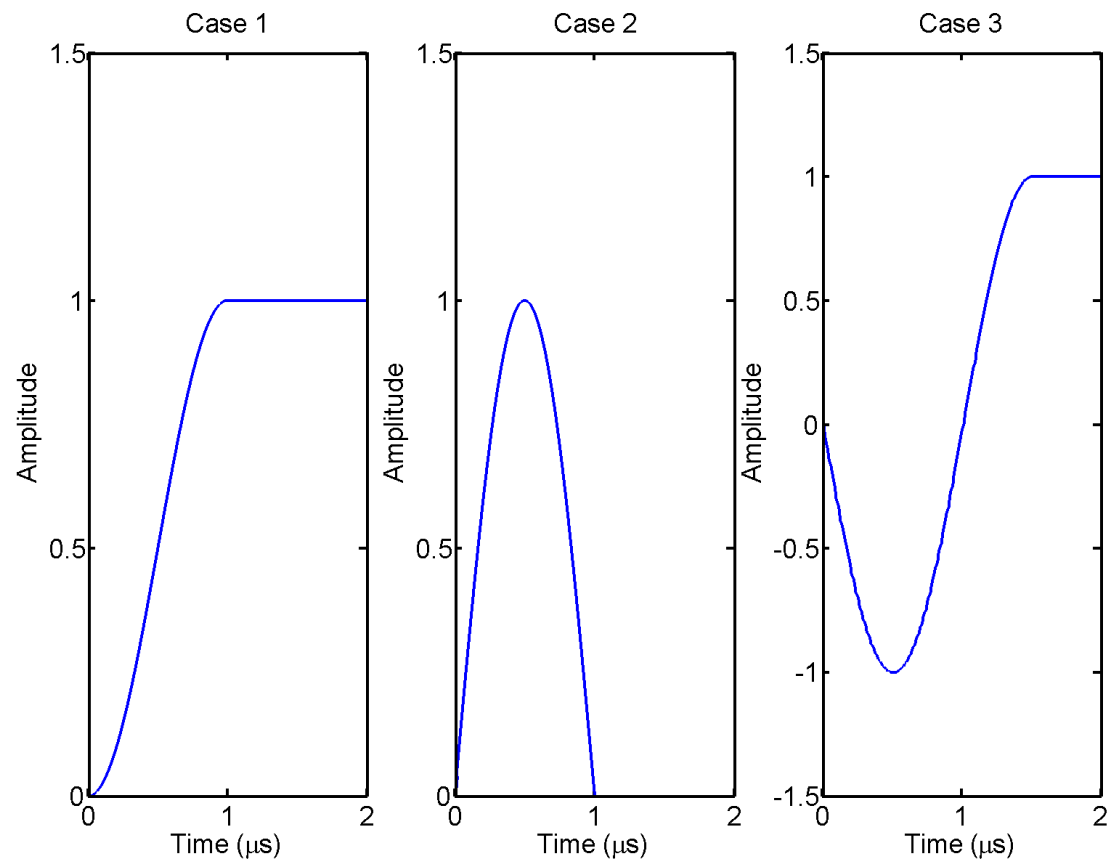

Figure 6. Three source-time functions used to simulate buried dipoles in an aluminum plate.

\section{III.B. Source Characterization}

In order to test the effectiveness of the source characterization algorithm described in section II.D, five AE sources were simulated using FEM. The outputs from these models were then analyzed and the moment tensor components and source-time functions were determined. First, the three dipoles simulated in figures 7-8 were analyzed. Next, a general 3-D source was modeled with a prescribed moment tensor and source-time function. Finally, a penny-shaped crack was modeled in an aluminum plate. The moment tensor and source time function were unknown for this case. 


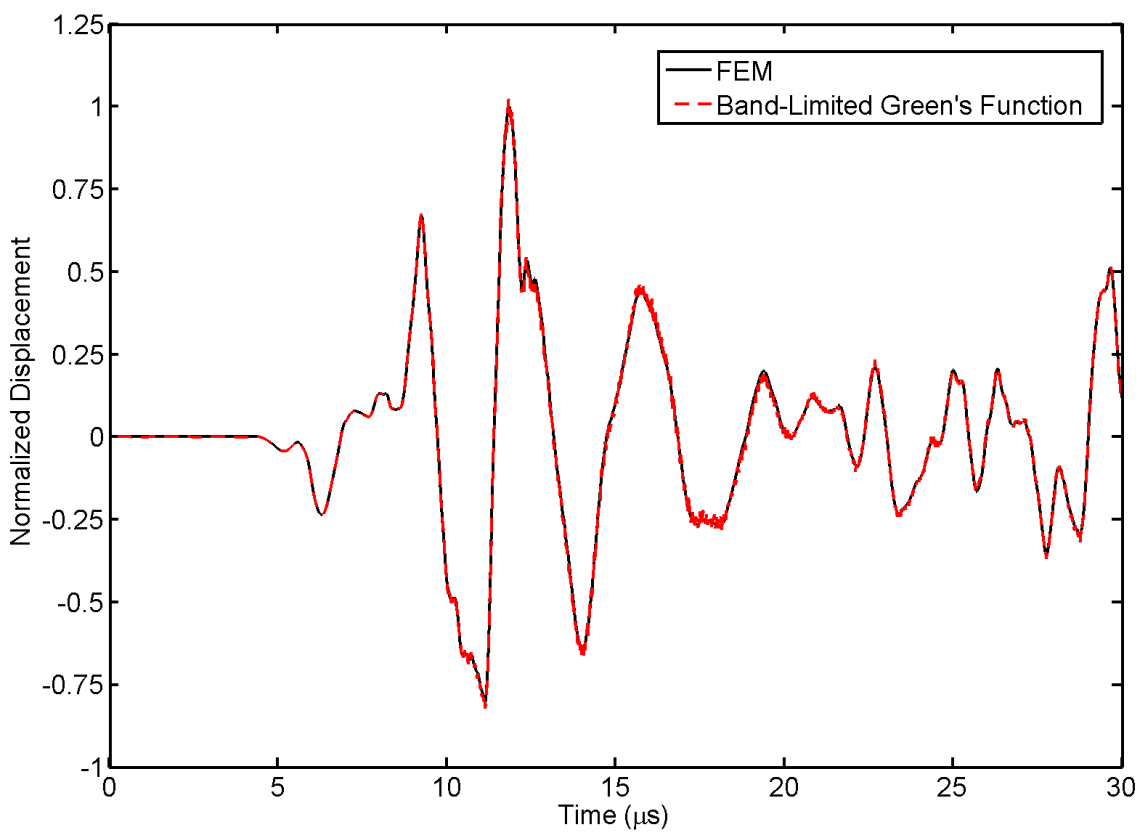

Figure 7. Case 1: Comparison between a finite element generated AE signal and the same signal calculated using the band-limited Green's function.

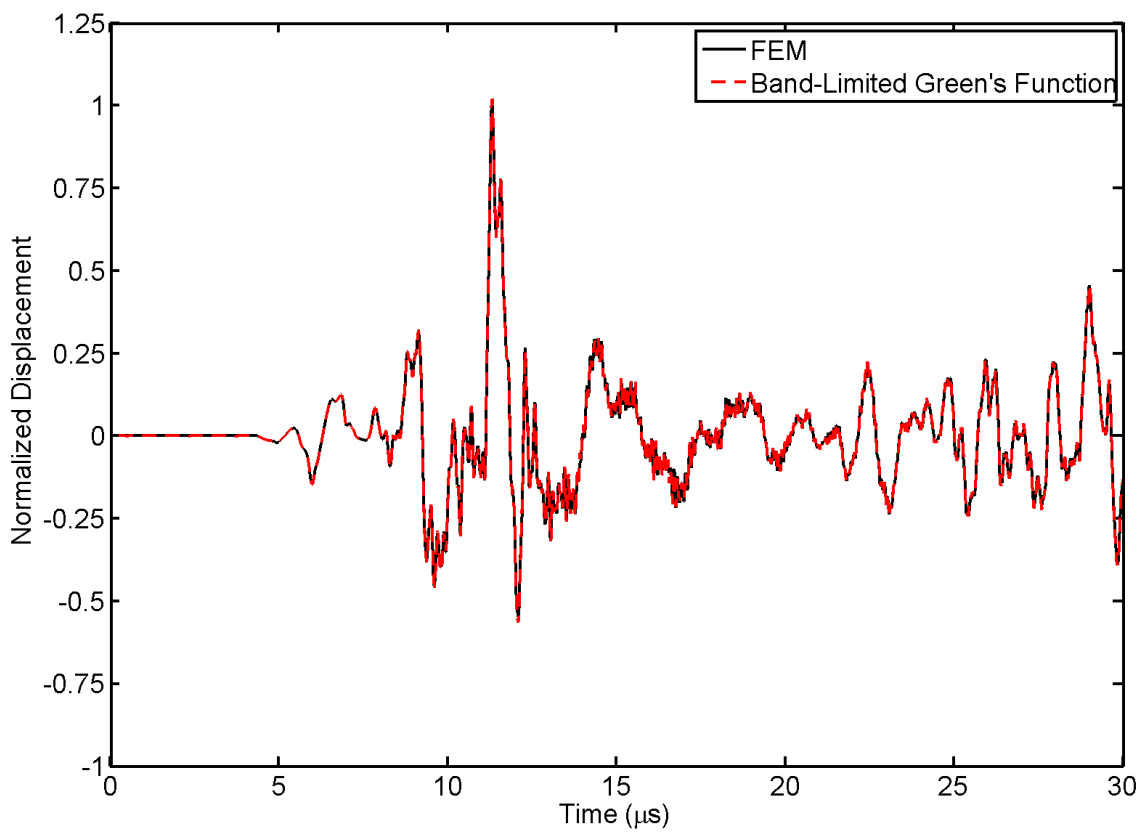

Figure 8. Case 2: Comparison between a finite element generated AE signal and the same signal calculated using the band-limited Green's function.

\section{III.B.1. Buried Dipoles}

The three dipoles were analyzed and the resulting calculated dipole strengths are shown in table 2 . The calculated source-time functions are shown in figure 10. The source-time functions found from the deconvolution procedure do not all have a peak value of 1 . The source-time function found for case 2 has a peak of 


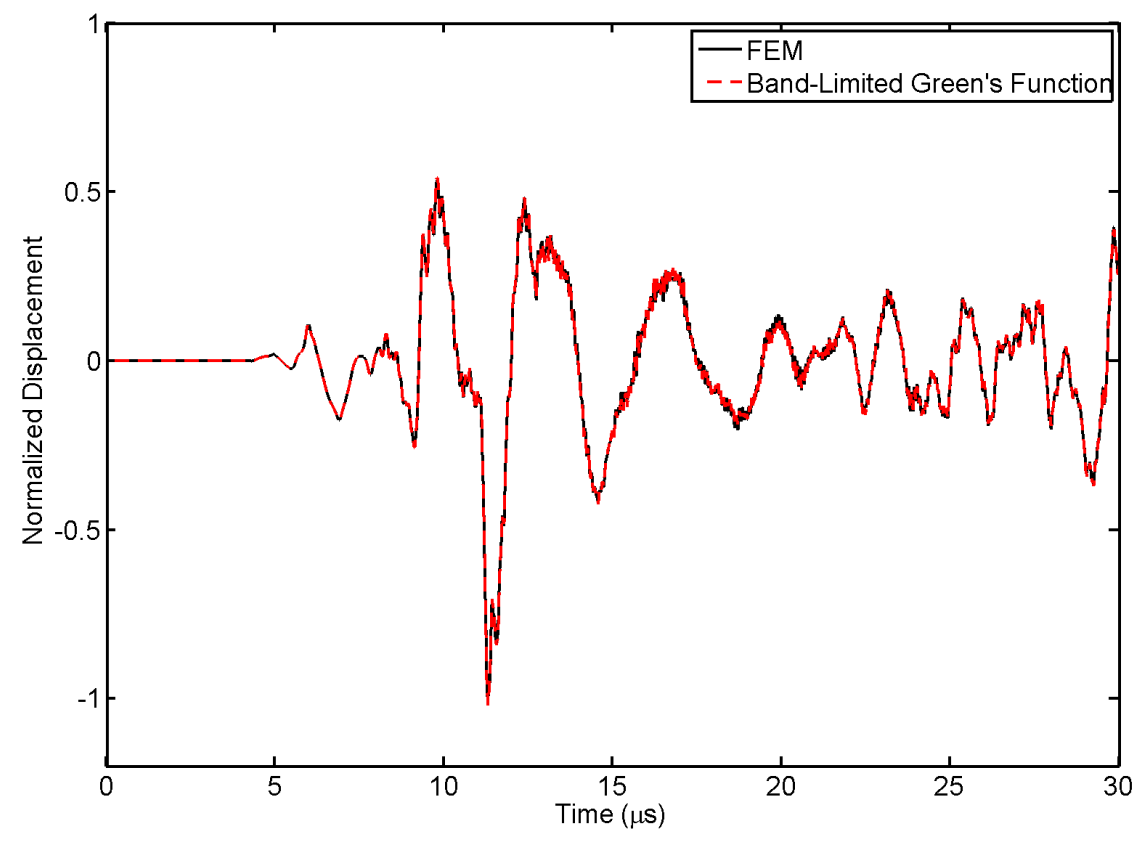

Figure 9. Case 3: Comparison between a finite element generated AE signal and the same signal calculated using the band-limited Green's function.

approximately 0.18 and case 3 peaks at 1.6. In both of these cases however, the recovered dipole strengths are such that when multiplied by the recovered source-time functions, the resulting peaks are close to the prescribed value of 0.3 . The calculated source-time functions normalized such that their peak values equal 1 are shown in figure 11. The corresponding normalized dipole strengths are then shown in the last row of table 2. The results match the prescribed values to within 7\%. It can also be seen that the recovered source-time functions do not settle out at 1 in case 1 and 3 , or 0 in case 2, but rather oscillate around these values. This is an artifact of the deconvolution procedure.

Table 2. Calculated dipole strengths

\begin{tabular}{lccc} 
& Case 1 & Case 2 & Case 3 \\
\hline Prescribed: & 0.30 & 0.30 & 0.30 \\
Calculated: & 0.31 & 2.00 & 0.17 \\
Normalized: & 0.31 & 0.28 & 0.28 \\
\hline \% Difference: & 3.3 & 6.6 & 6.6
\end{tabular}

\section{III.B.2. Three Dimensional Source}

A three-dimensional source was modeled in an aluminum plate as shown in figure 12. The source and sensor locations are shown in table 3. The prescribed moment tensor coefficients are listed in the first row of table 4 . The calculated source-time function was badly distorted due the deconvolution process, however, an estimate of the rise time (the time it takes the source function to reach an amplitude of 1 ) could be made. From this estimate, a refined initial guess was made that yielded a more accurate result. The prescribed source-time function along with the first pass and refined results are shown in figure 13. The source-time function as calculated on the first attempt (shown in cyan) reveals the general shape of the true source-time function: a rise time of around $0.9 \mu \mathrm{s}$ and constant force oscillating around an amplitude of 1 for $t>t_{\text {rise. }}$. Using this information, a refined initial guess for the source-time function was made, yielding the results shown in red which closely matches the prescribed source-time function. This demonstrates the sensitivity of the deconvolution algorithm to the initial guess supplied. The values of the calculated moment tensor coefficients 

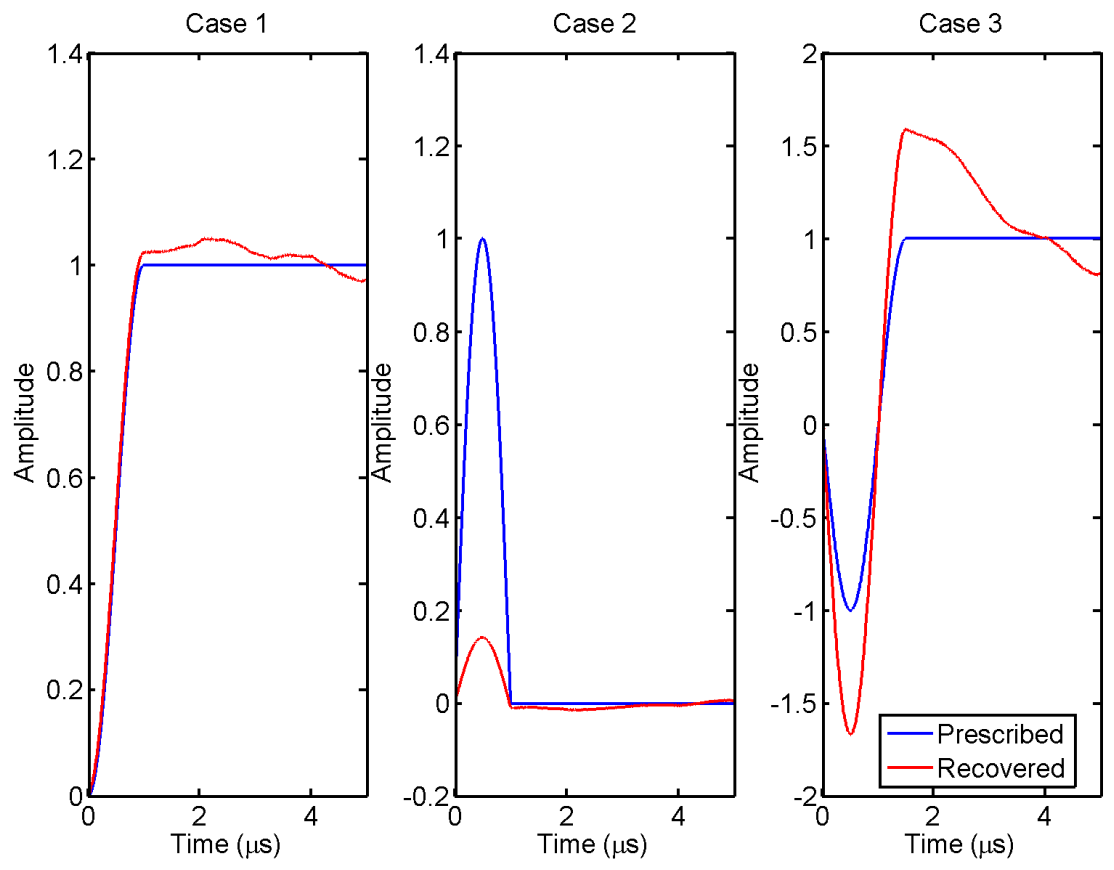

Figure 10. Comparison between prescribed and recovered source-time functions for three dipole sources.
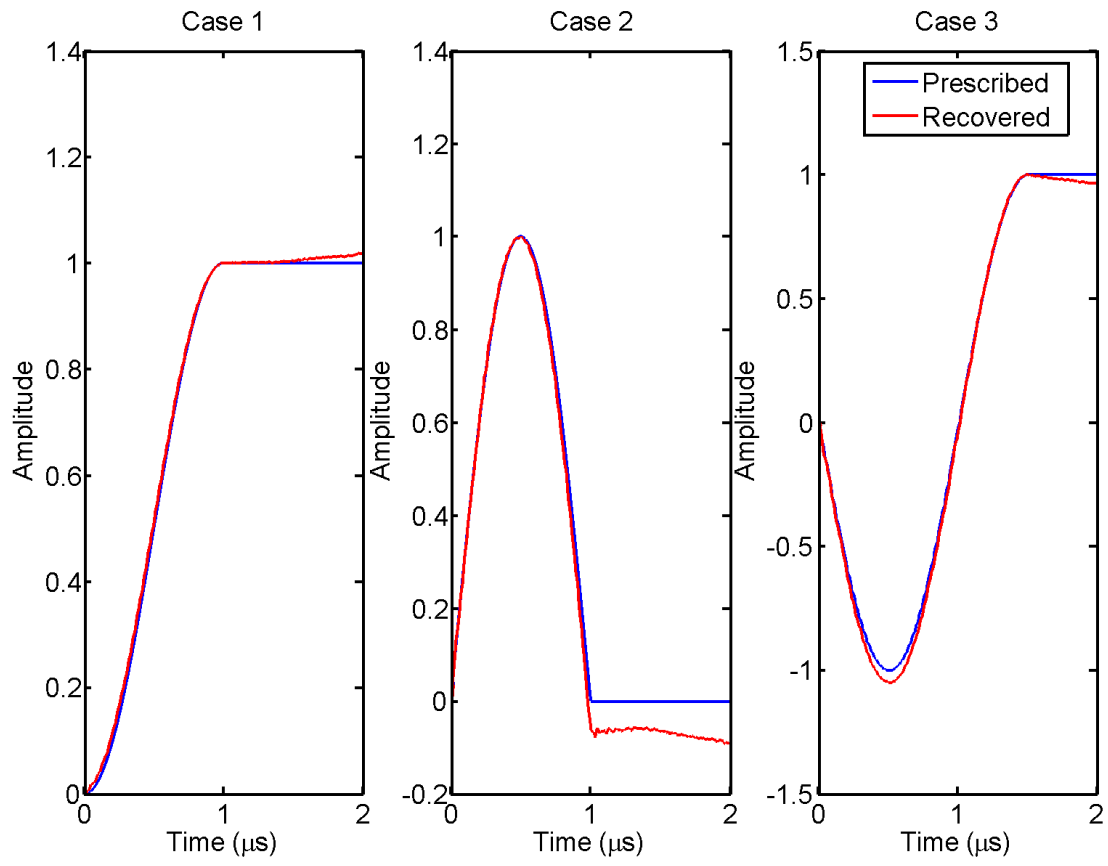

Figure 11. Comparison between prescribed and recovered source-time functions for three dipole sources after normalization of the recovered functions.

from the first pass and refined initial guess are shown in table 4 along with the $\%$ difference between the prescribed values and the final calculated values. The moment tensor coefficients were recovered to within $3 \%$. 


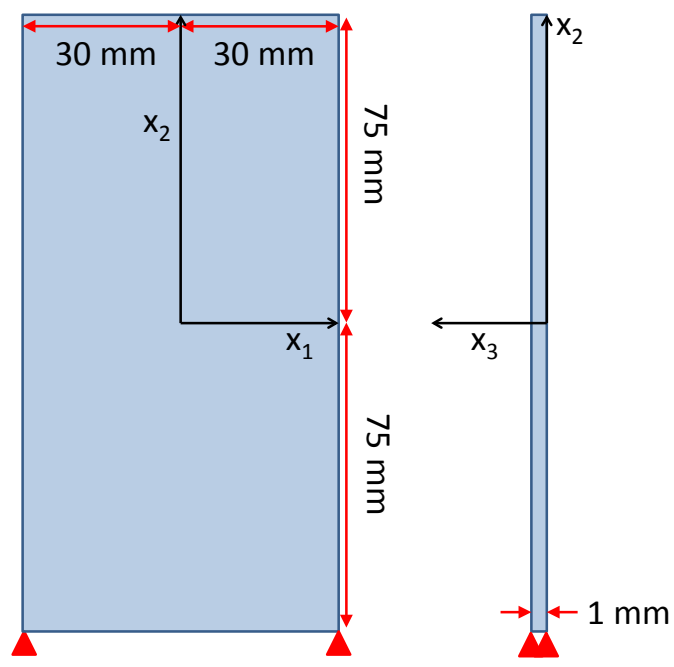

Figure 12. Schematic of geometry and boundary conditions for finite element model used for a general 3D source.

Table 3. Source and sensor locations for 3D source model

\begin{tabular}{lccc} 
& $x_{1}(\mathrm{~mm})$ & $x_{2}(\mathrm{~mm})$ & $x_{3}(\mathrm{~mm})$ \\
\hline Source: & 1.025 & 2.525 & 0.675 \\
Sensor 1: & -15.0 & 0.0 & 1.0 \\
Sensor 2: & 0.0 & 15.0 & 1.0 \\
Sensor 3: & 15.0 & 0.0 & 1.0 \\
Sensor 4: & 0.0 & -15.0 & 1.0 \\
Sensor 5: & -15.0 & 0.0 & 0.0 \\
Sensor 6: & 0.0 & 15.0 & 0.0 \\
Sensor 7: & 15 & 0.0 & 0.0 \\
Sensor 8: & 0.0 & -15.0 & 0.0 \\
Sensor 9: & -10.6 & 10.6 & 1.0 \\
Sensor 10: & -10.6 & 10.6 & 0.0 \\
Sensor 11: & 10.6 & 10.6 & 1.0 \\
Sensor 12: & 10.6 & 10.6 & 0.0 \\
Sensor 13: & 10.6 & -10.6 & 1.0 \\
Sensor 14: & 10.6 & -10.6 & 0.0 \\
Sensor 15: & -10.6 & -10.6 & 1.0 \\
Sensor 16: & -10.6 & -10.6 & 0.0
\end{tabular}

Table 4. Calculated moment tensor components for a three dimensional AE source

\begin{tabular}{lcccccc} 
& $m_{1}$ & $m_{2}$ & $m_{3}$ & $m_{4}$ & $m_{5}$ & $m_{6}$ \\
\hline Prescribed: & 0.40 & 1.35 & 0.75 & 0.00 & 0.00 & 0.00 \\
Calculated: & 0.33 & 1.30 & 0.58 & 0.00 & 0.00 & 0.00 \\
Refined: & 0.39 & 1.35 & 0.73 & 0.00 & 0.00 & 0.00 \\
\hline \% Difference: & 1.65 & 0.26 & 2.83 & 0.00 & 0.00 & 0.00
\end{tabular}

\section{III.B.3. Penny Shaped Crack}

A crack was modeled in a small aluminum plate, $2 \mathrm{~mm} \times 2 \mathrm{~mm} \times 1 \mathrm{~mm}$, as shown in figure 14 . The faces of the crack were held together while the specimen was loaded with a displacement boundary condition of 


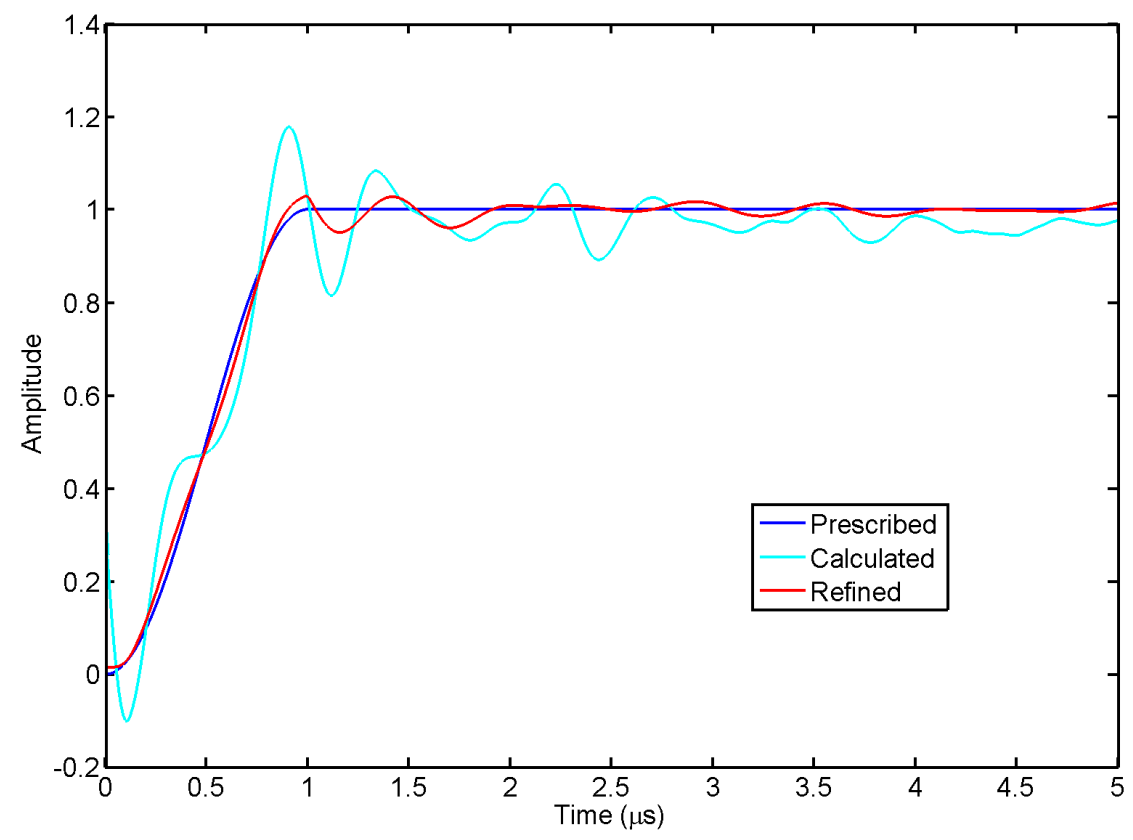

Figure 13. Comparison between an prescribed and recovered source-time function from a three dimensional AE source. The impact of an improved initial guess on the accuracy of the solution is demonstrated by the difference between the initial calculation shown in cyan and the refined calculation shown in red.

$0.01 \mathrm{~mm}$. After loading, the faces were released and the surface displacements from the sudden opening were recorded at 6 locations (listed in table 5 ). The signals were then analyzed. The recovered source-time function is shown in figure 15. The crack was found to have a rise time of approximately $0.3 \mu \mathrm{s}$ and the recovered moment tensor was

$$
M=\left[\begin{array}{ccc}
-1.73 e 10 & 5.75 e 10 & 0.02 e 10 \\
5.75 e 10 & -3.62 e 10 & -0.20 e 10 \\
0.02 e 10 & -0.20 e 10 & 1.21 e 10
\end{array}\right]
$$

The true source-time function and moment tensor are unknown for this source, therefore, in order to determine the accuracy of the solution, the recovered moment tensor and source-time function were used to reconstruct the signal using Eq. (11). The first $10 \mu s$ of the reference signal and the reconstruction are shown in figure 16. The percent difference between the samples in each signal was calculated. There was an average difference of $0.5 \%$ with a maximum difference of $6.36 \%$. The performance of the source characterization algorithm for the completely unknown source was comparable to the performance for the dipole and 3D source.

Table 5. Source and sensor locations for crack model

\begin{tabular}{lccc} 
& $x_{1}(\mathrm{~mm})$ & $x_{2}(\mathrm{~mm})$ & $x_{3}(\mathrm{~mm})$ \\
\hline Crack: & 0.0 & 0.0 & 0.5 \\
Sensor 1: & 0.0 & 0.5 & 1.0 \\
Sensor 2: & 0.0 & -0.5 & 1.0 \\
Sensor 3: & 0.5 & 0.0 & 0.0 \\
Sensor 4: & -0.5 & 0.0 & 0.0 \\
Sensor 5: & 1.0 & 0.0 & 0.5 \\
Sensor 6: & -1.0 & 0.0 & 0.5
\end{tabular}




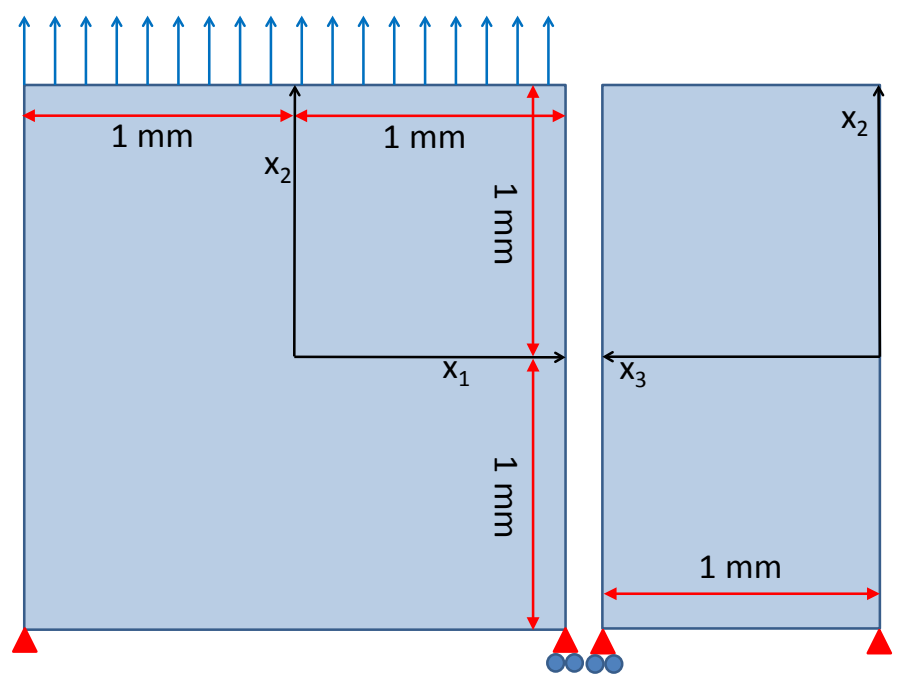

Figure 14. Schematic of geometry and boundary conditions for finite element model used to model a penny-shaped crack.

\section{Summary}

In this study, a method for estimating band-limited Green's functions using the finite element method is proposed. FEM is shown to be capable of accurately reproducing the displacement response to AE sources. Numerical examples were used to demonstrate the accuracy of the band-limited Green's functions. One FEM generated Green's function was used to calculate the response of 3 dipole sources with different temporal dependencies. The calculated responses were compared with FEM generated reference solutions.

An algorithm was developed to use the band-limited Green's functions to recover an unknown moment tensor and source-time function from an AE signal. Numerical examples were presented in which the sourcetime functions and moment tensors were extracted from FEM generated reference signals. In all cases, the rise times of the sources were precisely recovered. At times greater than the rise time, the recovered solutions tended to oscillate around the final amplitude of the source due to a build up of high frequency error during the deconvolution process. The moment tensor coefficients of the reference signals were recovered within $7 \%$ of the prescribed values. A moment tensor and source function were also recovered from a simulated crack. Future work will include experimental studies to improve the use of quantitative AE analysis techniques in structural health monitoring systems.

\section{References}

${ }^{1}$ Scruby, C. B., "An introduction to acoustic emission," Journal of Physics E: Scientific Instruments, Vol. 20, 1987, pp. 946-953.

${ }^{2}$ Scruby, C. B., "Quantitative acoustic emission techniques," Research Techniques in Nondestructive Testing, Vol. 8, 1985, pp. 141-210.

${ }^{3}$ Hsieh, P., Quantitative acoustic emission source characterization in an aluminum specimen, Ph.D. thesis, Cornell University, New York, 1987.

${ }^{4}$ Hamstad, M. A., O'Gallagher, A., and Gary, J., "Modeling of buried acoustic emission monopole and dipole sources with a finite element technique," Journal of Acoustic Emission, Vol. 17, No. 3-4, 1999, pp. 97-110.

${ }^{5}$ ABAQUS user's manual, version 6.11, Hibbit, Karlsson, and Sorensen, Inc., Pawtucket, RI, 2011.

${ }^{6}$ McLaskey, G. C. and Glaser, S. D., "Acoustic emission sensor calibration for absolute source measurements," Journal of Nondestructive Evaluation, Vol. 31, No. 2, 2012, pp. 157-168.

${ }^{7}$ Hestenes, M. R., "Methods of conjugate gradients for solving linear systems," Journal of Research of the National Bureau of Standards, Vol. 49, No. 6, 1952, pp. 409-436.

${ }^{8}$ Smith, S. W., The scientist and engineer's guide to digital signal processing, California Technical Publications, 1997. 


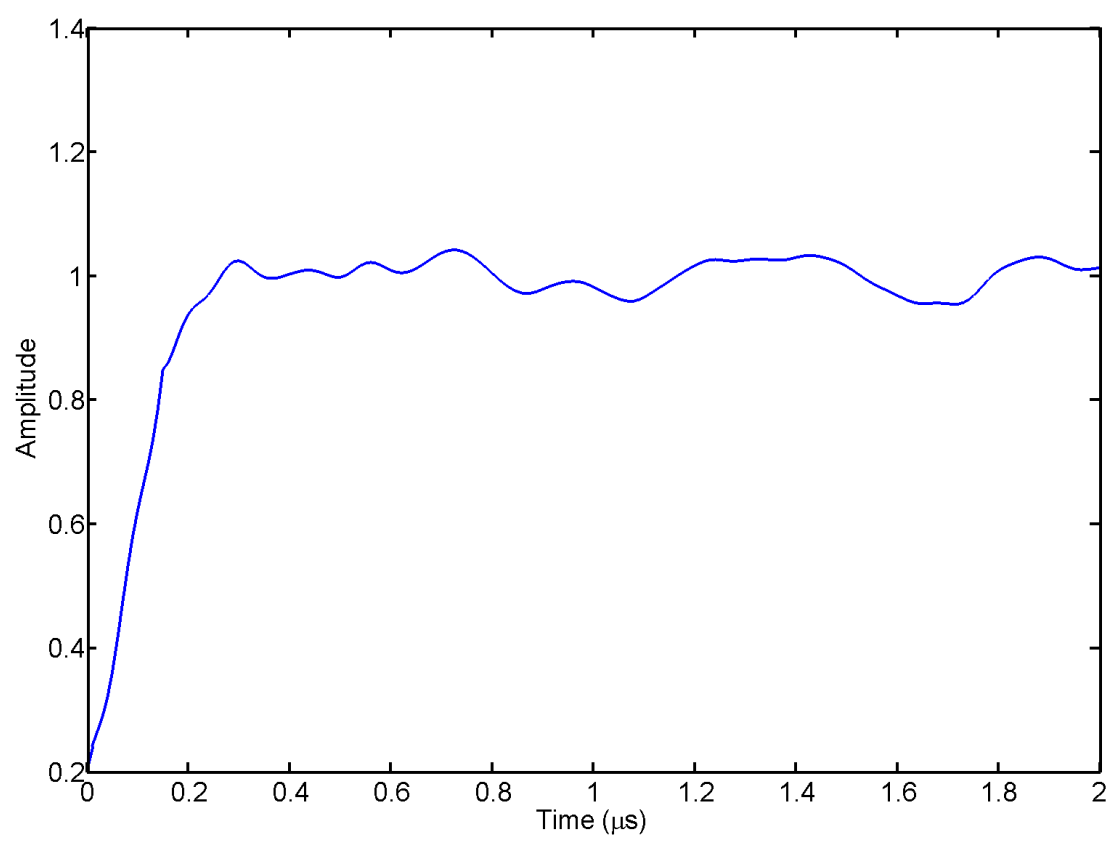

Figure 15. Recovered source-time function from the crack model.

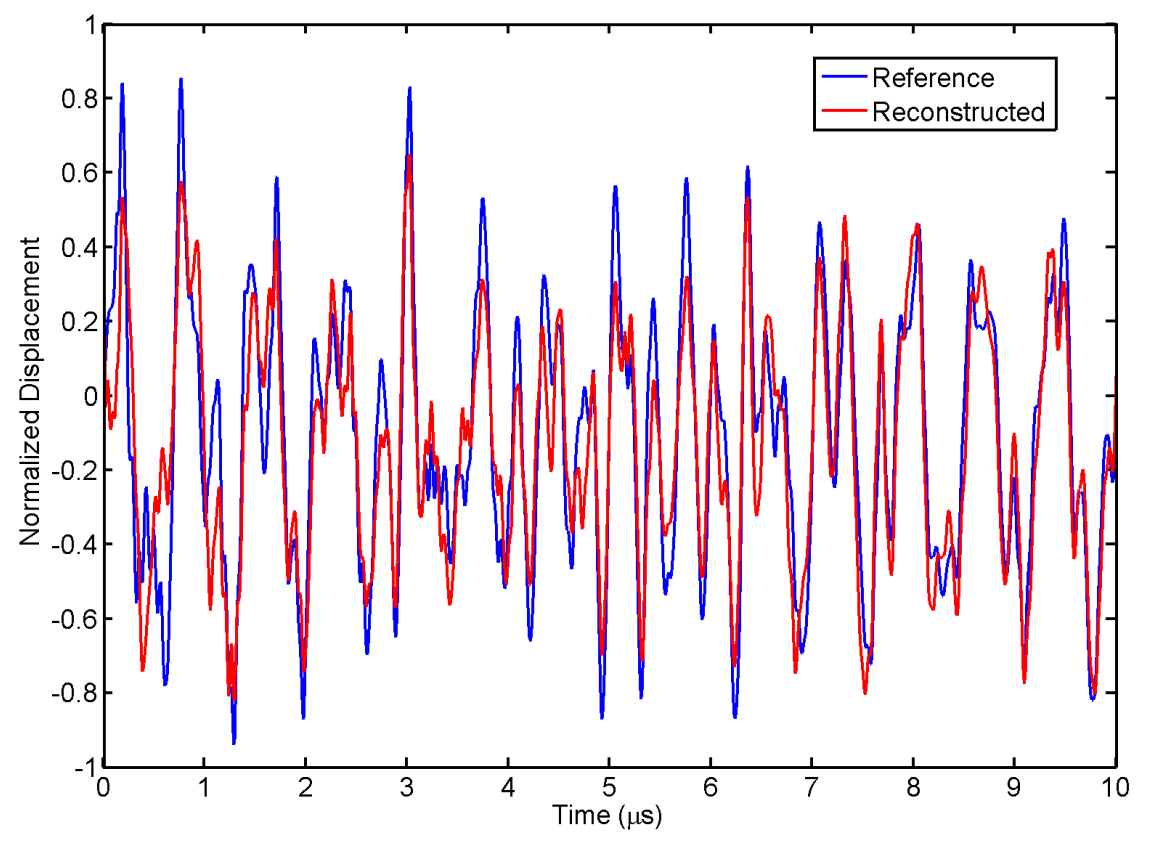

Figure 16. Comparison between the AE signal from the crack model and the reconstructed signal. 